\title{
The Role of Film Processing in the Large-Area Dielectric Breakdown Performance of Nano-Silica-BOPP Films
}

\author{
I. Rytöluoto ${ }^{1}$, K. Lahti $^{1}$, M. Ritamäki ${ }^{1}$ \& M. Karttunen ${ }^{2}$ \\ ${ }^{1}$ Tampere University of Technology, Department of Electrical Engineering, Tampere, Finland \\ ${ }^{2}$ Technical Research Centre of Finland, Tampere, Finland
}

\begin{abstract}
This paper summarizes the effects of various compositional, structural and film processing factors on the breakdown behavior of laboratory- and pilot-scale melt-compounded bi-axially oriented polypropylene (BOPP) nanocomposite films with silica fillers. A selfhealing multi-breakdown measurement approach has been extensively utilized for large-area breakdown characterization of a large number of material variants from different processing trials. The results suggest that although the optimum level of silica presumably resides at the low fill-fraction range $(\sim 1$ wt- $\%)$, the silica content itself is not the only determining factor, as compounds with equal silica content were found to exhibit large differences in the breakdown properties depending on the compounding and film processing steps. Dispersion quality and filler agglomeration (in both the nm- and $\mu \mathrm{m}$-scale) appear to be of great importance. Indications of possible interaction between nano-silica and co-stabilizer Irgafos 168 are also presented. Overall, the laboratory- and pilot-scale film processing trials suggest that up-scaling of the polymer nanocomposite production is sensible with traditional melt-blending technology, although further development and optimization of nanocomposite formulations and processing is necessary.
\end{abstract}

\section{Introduction}

In recent years, an increasing amount of research on dielectric polymer nanocomposite (PNC) films for nextgeneration high-energy-density capacitor applications has emerged and improvements in properties such as dielectric breakdown strength are strongly aspired for [1]. Inclusion of low amounts ( 1 wt-\%) of permittivitymatching nanoparticles such as silica in a suitable polymer matrix has been shown to be a promising approach, and some improvements in the short-term dielectric strength and breakdown distribution homogeneity have been previously reported [2]-[4]. However, as the dielectric breakdown performance of polymer-based thin films is largely dependent on various processing- and material-related aspects [5], [6], a thorough understanding of the underlying factors and optimization of the film processing parameters are crucial in order to achieve improved breakdown strength in practical insulation systems. Furthermore, provided that a potential PNC formulation is developed, it is equally important to consider whether or not it is possible to up-scale the film production process towards large-scale industrial manufacturing.
Our research has previously been focused on the DC breakdown performance of melt-compounded $\mathrm{SiO}_{2}$ BOPP PNC films. A large-area multi-breakdown measurement approach based on the self-healing breakdown capability of metallized polymer films has been extensively applied for studying the breakdown properties, as it enables a large amount of breakdown data to be acquired from a relatively large total film area hence leading to a high statistical accuracy even at low breakdown probabilities. A detailed description and evaluation of the measurement method was recently given in [7] while studies exemplifying the large-area multi-breakdown characterization of various laboratoryscale, pilot-scale and commercial BOPP and BOPPPNC films are presented in [3], [4], [8], [9].

The purpose of this paper is to make an overview of various material- and processing-related factors and their effects on the large-area multi-breakdown performance of $\mathrm{SiO}_{2}$-BOPP PNC films. Special emphasis is put on the compositional and processing features which considerably affect the breakdown properties. Furthermore, up-scaling aspects are discussed and breakdown properties of $\mathrm{SiO}_{2}-\mathrm{BOPP}$ PNC films produced on a Brückner pilot film line are compared with laboratory-scale films.

\section{Experimental}

\subsection{Materials and film processing}

A number of $\mathrm{SiO}_{2}$-BOPP PNC film production trials were carried out in order to study the effects of various processing parameters on the breakdown performance. The studied processing trials were:
1. Effect of nano-SiO $\mathrm{S}_{2}$ fill-fraction
2. Effect of antioxidants
3. Effect of pre-mixing technique
4. Effect of compounder output and screw speed
5. Effect of bi-axial orientation temperature
6. Pilot-scale film production

Figure 1 presents an overview of the film processing steps together with the processing parameters which were varied along the production chain. The film processing was conducted at the VTT Technical Research Centre of Finland and at a Brückner pilot film line in Denmark. As the total amount of studied compounds was over 25, a detailed listing of all the materials, processing parameters and film specifications is out of the scope of this paper and can be found from the related publications ([3], [4], [7]). Only the essential aspects are summarized in the following. 


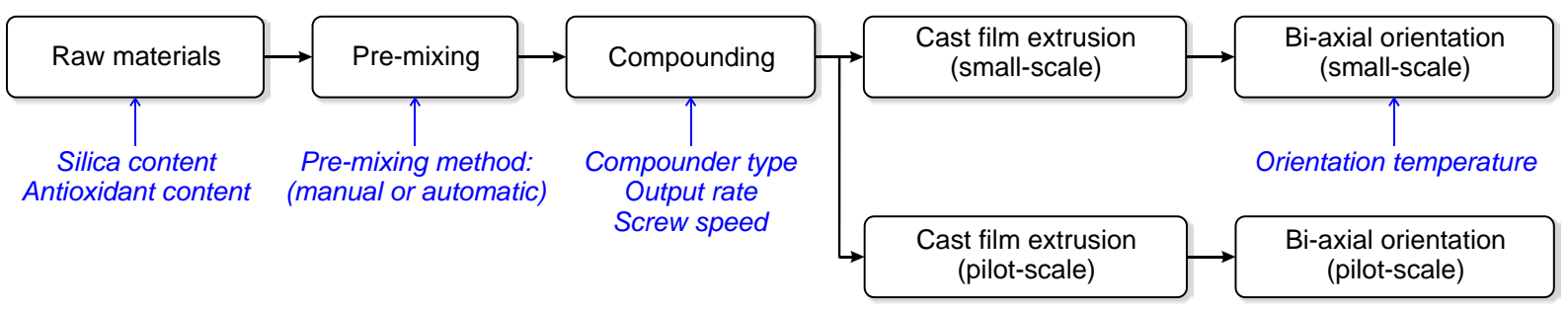

Figure $1-\mathrm{SiO}_{2}$-BOPP PNC film processing steps and studied variables. (black = processing step, blue = studied parameter)

Unstabilized polypropylene (PP) homopolymer HC318BF in powder form (a non-commercial product from Borealis N.V.) was used as the matrix polymer. Nanofiller content of $4.5 \mathrm{wt} \%$ of hexamethyldisilazane (HMDS)-treated hydrophobic fumed nano-silica (Aerosil R $812 \mathrm{~S}$ by Evonik) was used in most of the compounds, however, lower silica contents of 1.0 and $2.0 \mathrm{wt}-\%$ were also studied in one of the trials. Process stabilizer Irganox 1010 (0.47 wt-\%) and co-stabilizer Irgafos 168 (0.35 wt-\%) were added to the compounds in order to prevent thermo-oxidative degradation of PP during melt-processing. The co-stabilizer content was varied in one of the trials ( $0-0.35 \mathrm{wt}-\%)$.

For the laboratory-scale films, the general film processing steps were as follows. Initially, the raw materials (in powder form) were pre-mixed either manually in polyethylene bag or by using various automatic mixers and thereafter compounded mainly by a Berstorff ZE 25/48D twin screw compounder with high-shear screw geometry, two separate kneading sections and a melt filter (screen size $42 \mu \mathrm{m}$ ). The screw speed and output of the Berstorff ZE 25/48D compounder were $300 \mathrm{rpm}$ and $9 \mathrm{~kg} / \mathrm{h}$, respectively, except for one trial in which the output was varied (3$15 \mathrm{~kg} / \mathrm{h}$ at $300 \mathrm{rpm})$. Additionally, a high-speed twin screw Berstorff ZE 25 x 49D UTX compounder and a varying screw speed was also used in one of the trials (output $8 \mathrm{~kg} / \mathrm{h}$ ). The compounded strands were cooled and pelletized and thereafter extruded into $\sim 500 \mu \mathrm{m}$ cast films by a Brabender Plasticorder single screw extruder with a three-layer screen pack. Finally, the cast films were bi-axially oriented with a Brückner KARO IV film stretching machine (set temperature mainly $157^{\circ} \mathrm{C}$, stretching ratio $5.4 \times 5.4$ ), resulting in BOPP films $~ 15-$ $23 \mu \mathrm{m}$ in thickness. Detailed compounder and cast extrusion temperatures etc. can be found from [3].

Some of the compounds were also chosen for a pilot-scale film trial. The compounds (100-140 kg) were prepared similarly as described above. Automatic pre-mixing and the Berstorff ZE 25/48D compounder were used for the compounds presented in this paper. Pilot-scale films were then produced at a Brückner pilot film line in Denmark consisting of a single screw extruder with a flat die, a casting station, machine- and transverse direction orientation (MDO and TDO) units and a winder. The polymer compound was first melted in the extruder, quenched on the casting roll and then transferred to the MDO where it was reheated and stretched in the machine direction. Thereafter, the film was reheated in the TDO unit before transverse stretching and cooled down before winding.

\subsection{Large-area multi-breakdown measurement and breakdown data analysis}

Sample films for the multi-breakdown measurement were cut to $11 \times 11 \mathrm{~cm}^{2}$ dimensions from the bi-axially oriented film sheets. Thorough film thickness mapping was seen necessary in order to avoid error in the breakdown field calculation later on. Therefore, sample film thicknesses were measured systematically at 25 points covering a $10 \times 10 \mathrm{~cm}^{2}$ area with LE1000-1 highprecision thickness measurement gauge (accuracy 0.1 $\mu \mathrm{m}$, resolution $0.05 \mu \mathrm{m})$. In order to realize the selfhealing breakdown capability, Zn-Al-metallized BOPP film (12 $\mu \mathrm{m}$ Tervakoski PSX) was used as the electrode film. The sample film was sandwiched between two electrode films (metallized surfaces facing towards the sample film) and two additional sheets of $100 \mu \mathrm{m}$ thick transparent polyester film in order to provide mechanical support, thus forming a test capacitor with an active area of $81 \mathrm{~cm}^{2}$. The whole film arrangement was then fastened to a test bench consisting of a Bakelite bottom plate and a transparent acrylic plastic top plate. Electrical contacts with the metallized films were made by means of aluminum clamp electrodes at the both ends of the bottom plate. Six sample films were measured per each material resulting in $486 \mathrm{~cm}^{2}$ total film area.

The measurements were conducted in insulating oil (Shell Diala DX) in accordance with IEC-60243 standard in order to mitigate surface flashovers. The DC voltage across the test capacitor was first raised to approximately $40-60 \%$ of the probable short-time breakdown voltage with a fast ramp speed of $400 \mathrm{~V} / \mathrm{s}$, after which a slow ramp speed of $30 \mathrm{~V} / \mathrm{s}$ was used for measuring the self-healing breakdown events, roughly in accordance to the slow rate-of-rise test defined in the IEC-60243-1. The test setup and the procedure are discussed in more detail in [7].

During each discharge event in the test capacitor, the discharge current, test capacitor voltage and the timesignature of the event were recorded with a highresolution oscilloscope operated in the sequenceacquisition mode and triggered to the positive rising edge of the discharge current signal. This allowed detailed determination of the breakdown voltage, voltage drop, peak current, discharge energy and various pulse time parameters for each event. A video was recorded from the top of the test capacitor unit for the whole duration of the breakdown measurement, allowing detailed chronological analysis of the breakdown progression of the sample after the 
measurement. Breakdown fields were determined manually by rigorously determining the average thickness around the discharge spot (by means of the video recording) and by calculating the average breakdown field event-by-event in MATLAB.

Prior to the statistical analysis, a data selection procedure based on the discharge energy characteristics of the self-healing breakdown process was utilized in order to exclude non-breakdown events from the measurement data (such as partial discharges and consecutive breakdowns occurring close to or at previous breakdown sites). The breakdown data selection was performed in MATLAB. Detailed description of the procedure is omitted here and can be found elsewhere [7], [8]. Finally, for the statistical analysis of the qualified breakdown data sets, single 2parameter Weibull distributions and, if necessary, additively mixed 2-parameter Weibull distributions were utilized:

$$
F(x)=\sum_{i=1}^{S} \frac{N_{i}}{N} F_{i}(x)=\sum_{i=1}^{S} \frac{N_{i}}{N}\left[1-\exp \left\{-\left(\frac{x}{\alpha_{i}}\right)^{\beta_{i}}\right\}\right] .
$$

In Eq. (1), $F_{i}(x)$ is the 2-parameter Weibull distribution, $S$ is the number of subpopulations, $N_{i} / N$ is the portion (\%) of the subpopulation $i$ and $\alpha_{i}$ and $\beta_{i}$ are the Weibull parameters of subpopulation $i$. The scale parameter $\alpha$ corresponds to the value of $x$ at the $63.2 \%$ failure probability and the shape parameter $\beta$ depicts the slope or homogeneity of the fitted distribution. In the case of a single 2-parameter Weibull distribution, $S$ and the portion $N_{i} / N$ in Eq. (1) are equal to 1 and $100 \%$, respectively. Maximum-likelihood estimation (MLE) and non-linear regression (NLR) methods were used for the parameter estimation and the analysis was performed with Weibull++ and MATLAB software. $90 \%$ confidence bounds were calculated for the Weibull parameters and breakdown percentiles by the Fisher Matrix (FM) method.

\subsection{Nanoparticle size and dispersion quality analysis}

Samples of the $\mathrm{SiO}_{2}$-BOPP films were broken in liquid nitrogen and the cross-sectional surface morphologies were imaged with a JEOL JSM-6360LV scanning electron microscope (SEM). The SEM images were converted to binary images and the particle area distributions were determined by using ImageJ software. Moreover, a parameter describing the characteristic length of the unreinforced polymer domains, free-space length $L_{f}$, was also calculated and used to quantify the dispersion quality [3], [10]. $L_{f}$ is defined as the width of the largest randomly placed square for which the most probable number of intersecting particles is zero. The MATLAB code for calculating $L_{f}$ was retrieved and used according to the literature [10]. Further details about the free-space length characterization and its interpretation are available from the above publications.

\section{Results and discussion}

\subsection{Large-area multi-breakdown results}

Figure 2 presents a set of large-area multi-breakdown distributions from various processing trials on the Weibull probability scale along with the Weibull fits calculated according to Eq. (1). If applicable, single 2parameter Weibull distributions were fitted to the breakdown data; otherwise mixed Weibull distributions were utilized on the basis of the best goodness-of-fit test result. $90 \%$ confidence bounds were also calculated for each data set but they are omitted from Figure 2 for the sake of clarity.

As also discussed in [3], [4], Figure 2a clearly shows that an increase in silica content reduces the breakdown performance in the $>5 \%$ breakdown probability region in comparison to the unfilled reference BOPP but also leads to a concurrent improvement of the breakdown distribution homogeneity. Interestingly, the Weibull lines of the $1.0 \mathrm{wt}-\% \mathrm{SiO}_{2}$-BOPP PNC and the unfilled BOPP intersect in the 5-10\% breakdown probability range with the 1.0 wt- $\% \mathrm{SiO}_{2}$-BOPP PNC showing higher strength in the low probability region. This is promising, as considering film capacitors, for which the operative electric field is mandated by the electrical weak points in the polymer film, both the increase of breakdown distribution homogeneity (Weibull $\beta$ ) and the improvement in the low-probability breakdown behavior are substantially more relevant than the breakdown behavior in the higher $(>10 \%)$ breakdown probability region.

It is remarked that transmission electron microscope (TEM) imaging revealed micron-sized agglomerates in the $\mathrm{SiO}_{2}-\mathrm{PP}$ cast films [3] which most likely impact the breakdown performance. While the compounds presented in Figure 2a were pre-mixed manually, the possible effect of automatic mixing equipment on the dispersion quality was studied in another trial with 4.5 wt-\% $\mathrm{SiO}_{2}$-BOPP compounds (see [3]). Figure 3a-b present exemplifying SEM cross-sectional images of the films from the pre-mixing trial. Although pre-mixing technique appears to affect the silica nanodispersion, the multi-breakdown measurements suggested that the premixing technique had little or no effect on the breakdown behavior. This, conversely, also confirms that automatic pre-mixing of larger batches is applicable for up-scaling purposes.

Figure $2 b$ presents a set of previously unpublished results from another trial in which a high-speed twin screw Berstorff ZE 25 x 49D UTX compounder with a varying screw speed (300, 600 and $1200 \mathrm{rpm}$ ) was used for compounding 4.5 wt- $\% \mathrm{SiO}_{2}$-PP. SEM crosssectional images and the corresponding particle analysis results of the above films are presented in Figure 3c-e. Surprisingly, the 4.5 wt- $\% \mathrm{SiO}_{2}$-BOPP compounded with the slowest (300 rpm) screw speed shows a considerable shift towards higher dielectric strength while the 600 and 1200 rpm variants reside in the same range as the $4.5 \mathrm{wt}-\% \mathrm{SiO}_{2}$-BOPP presented earlier in 
Figure 2a. For further comparison, Figure $2 \mathrm{~b}$ also shows the 1.0 wt-\% $\mathrm{SiO}_{2}$-BOPP from Figure 2a, which exhibits a breakdown distribution strikingly similar to that of the 4.5 wt-\% $\mathrm{SiO}_{2}$-BOPP compounded with the $300 \mathrm{rpm}$ screw speed. This result suggests that the silica wt-\% content alone is not the only determining factor for $\mathrm{SiO}_{2}$-BOPP PNC breakdown performance. Speculative explanations for the exceptional behavior could be e.g. improvement in silica dispersion and distribution or reduction of silica agglomeration with slower compounding. Although Figure 3c-e suggest no major differences in the nano-scale dispersion qualities or particle sizes, it is reminded that a large number of SEM images would be required to obtain a statistically reliable estimate of the whole particle size distribution in the materials. While probably scarce in quantity, larger agglomerates may hinder the otherwise beneficial effect of good nanodispersion [11]. Indeed, a few

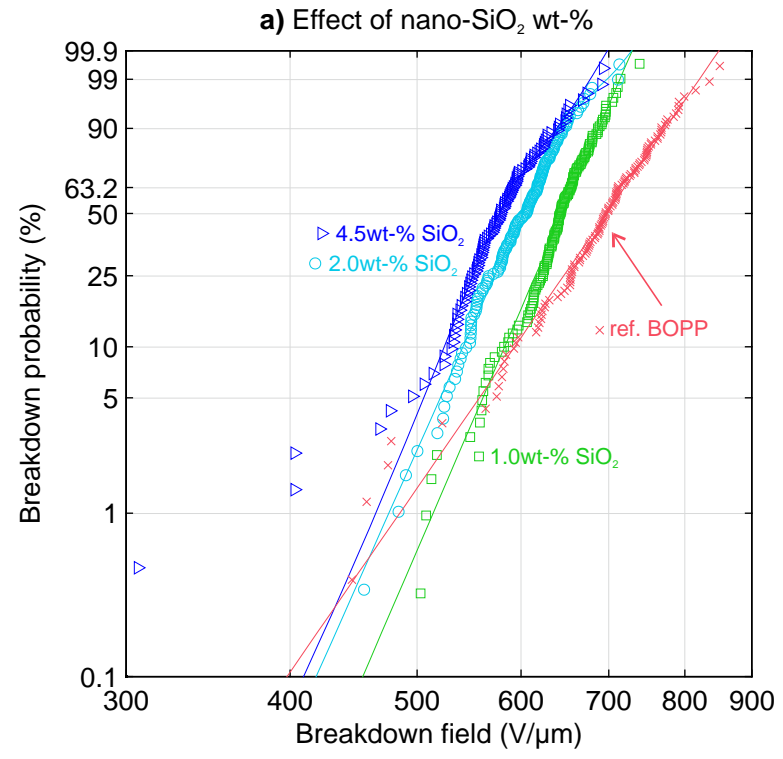

c) Effect of co- stabilizer content (Irgafos 168)

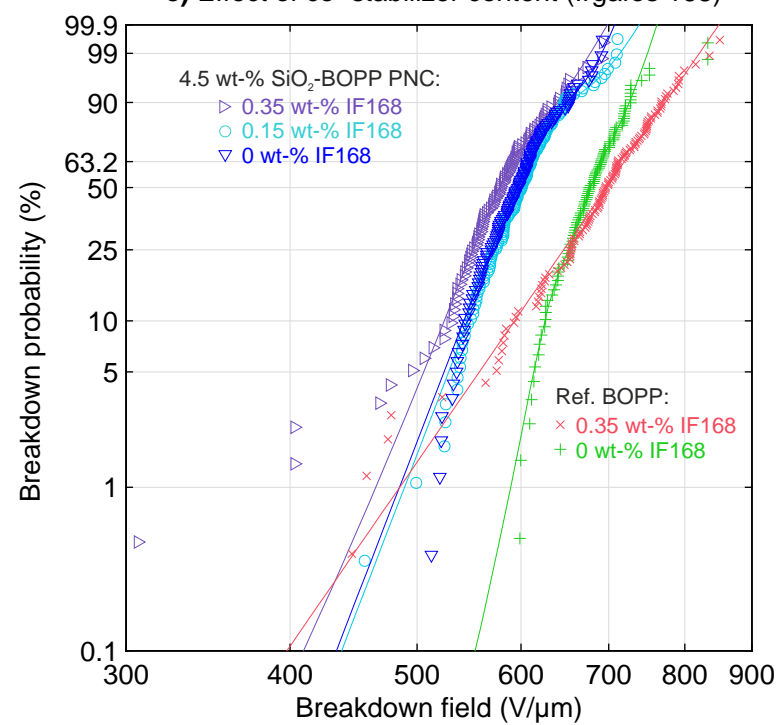

agglomerates 500 $\mathrm{nm}$ in diameter were observed in some of the SEM images of the $1200 \mathrm{rpm} 4.5 \mathrm{wt}-\%$ $\mathrm{SiO}_{2}$-BOPP compound. On the other hand, another explanation for the results could be related to differences in antioxidant depletion rate with different compounding rates. It is remarked that residual antioxidant content in BOPP films (e.g. the main process stabilizer Irganox 1010) has been shown to impair the breakdown strength [12], [13]. A more detailed analysis is however out of the scope of this paper.

The effect of varying co-stabilizer (Irgafos 168) content on the breakdown performance is presented in Figure 2c. The unfilled BOPP shows a drastic difference in breakdown distribution homogeneity depending on whether Irgafos 168 was used or not, indicating that Irgafos 168 has a detrimental effect on the breakdown performance of the end-product. Interestingly, the

b) Effect of compounder screw speed

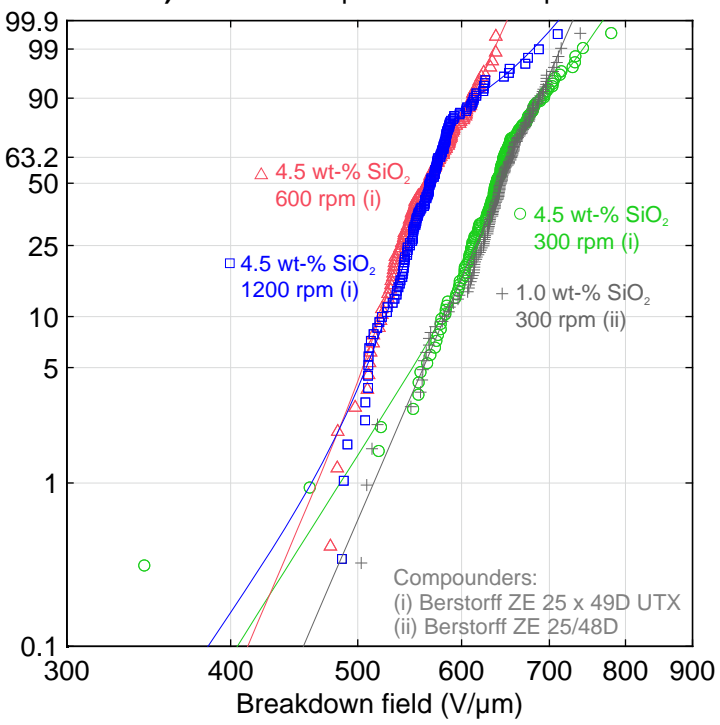

d) Laboratory- vs. pilot-scale films

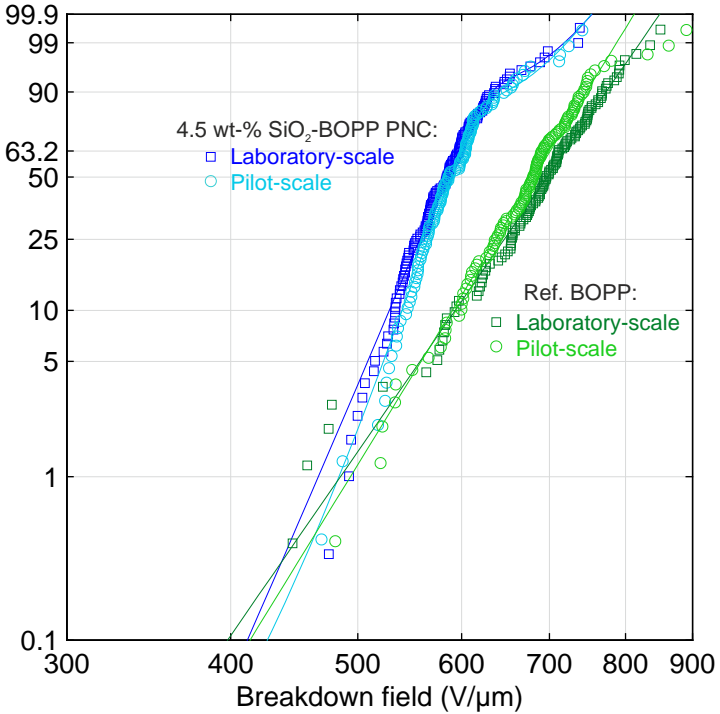

Figure 2 - Large-area multi-breakdown performance of $\mathrm{BOPP}-\mathrm{SiO}_{2}$ and reference-BOPP films. The Weibull probability plots show the effects of a) nano-silica filler content, b) compounder screw speed, c) co-stabilizer (Irgafos 168) content and d) a comparison of laboratory- and pilot-scale films. 6 samples $\left(486 \mathrm{~cm}^{2}\right.$ total film area) per material were measured. $90 \%$ confidence bounds were calculated but are not shown for clarity. 
dependence on Irgafos 168 content is less prominent with the 4.5 wt-\% $\mathrm{SiO}_{2}$-BOPP variants which points towards possible interaction mechanism between silica and antioxidants. For example, a study by Nawaz et al. [14] suggests that a substantial amount of antioxidants can be adsorbed on the filler surfaces. Initial antioxidant adsorption on the nanofiller surfaces, film processing and the possible slow-release of the antioxidant residue from the adsorption sites over time may have an influence on both the short- and long-term breakdown performance of the end-product and should be investigated further in the future.

a)

$4.5 \mathrm{wt}-\% \mathrm{SiO}_{2}$-BOPP Automatic pre-mixing Berstorff ZE 25/48D $300 \mathrm{rpm}$ $L_{f}=563 \mathrm{~nm}$

b)

4.5 wt- $\% \mathrm{SiO}_{2}-\mathrm{BOPP}$ Manual pre-mixing Berstorff ZE 25/48D $300 \mathrm{rpm}$ $L_{f}=352 \mathrm{~nm}$

c)

4.5 wt- $\% \mathrm{SiO}_{2}$-BOPP Automatic pre-mixing Berstorff ZE 25 x 49D UTX $300 \mathrm{rpm}$ $L_{f}=596 \mathrm{~nm}$

d)

4.5 wt- $\% \mathrm{SiO}_{2}$-BOPP Automatic pre-mixing Berstorff ZE 25 x 49D UTX $600 \mathrm{rpm}$ $L_{f}=617 \mathrm{~nm}$

\section{e)}

4.5 wt- $\% \mathrm{SiO}_{2}-\mathrm{BOPP}$ Automatic pre-mixing Berstorff ZE 25 x 49D UTX $1200 \mathrm{rpm}$ $L_{f}=617 \mathrm{~nm}$
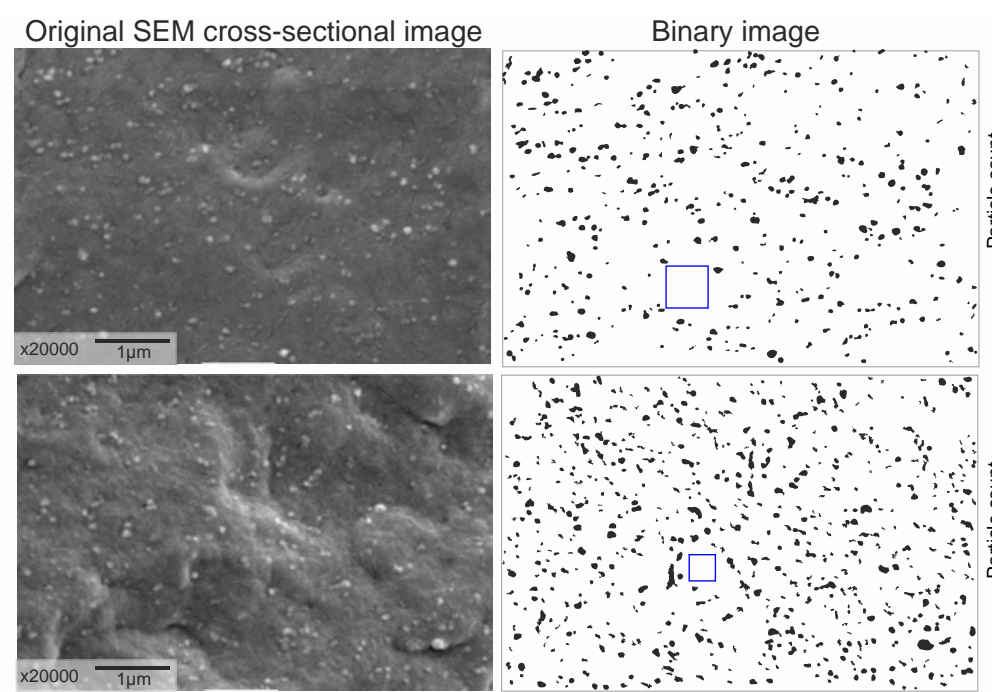

Lastly, Figure 2d presents a comparison of laboratory- and pilot-scale 4.5 wt-\% $\mathrm{SiO}_{2}$-BOPP PNC films with the corresponding unfilled reference films. It is evident that the up-scaling of the compounding and film processing stages was successful and resulted in films with similar breakdown properties as the corresponding laboratory-scale variants, in spite of the differences in the cast extrusion and bi-axial orientation steps (see [3]). Although more work is certainly needed to develop and optimize the PNC formulation, the results suggest that PNC film production for capacitor applications using traditional melt-blending technology

Binary image Particle area histogram
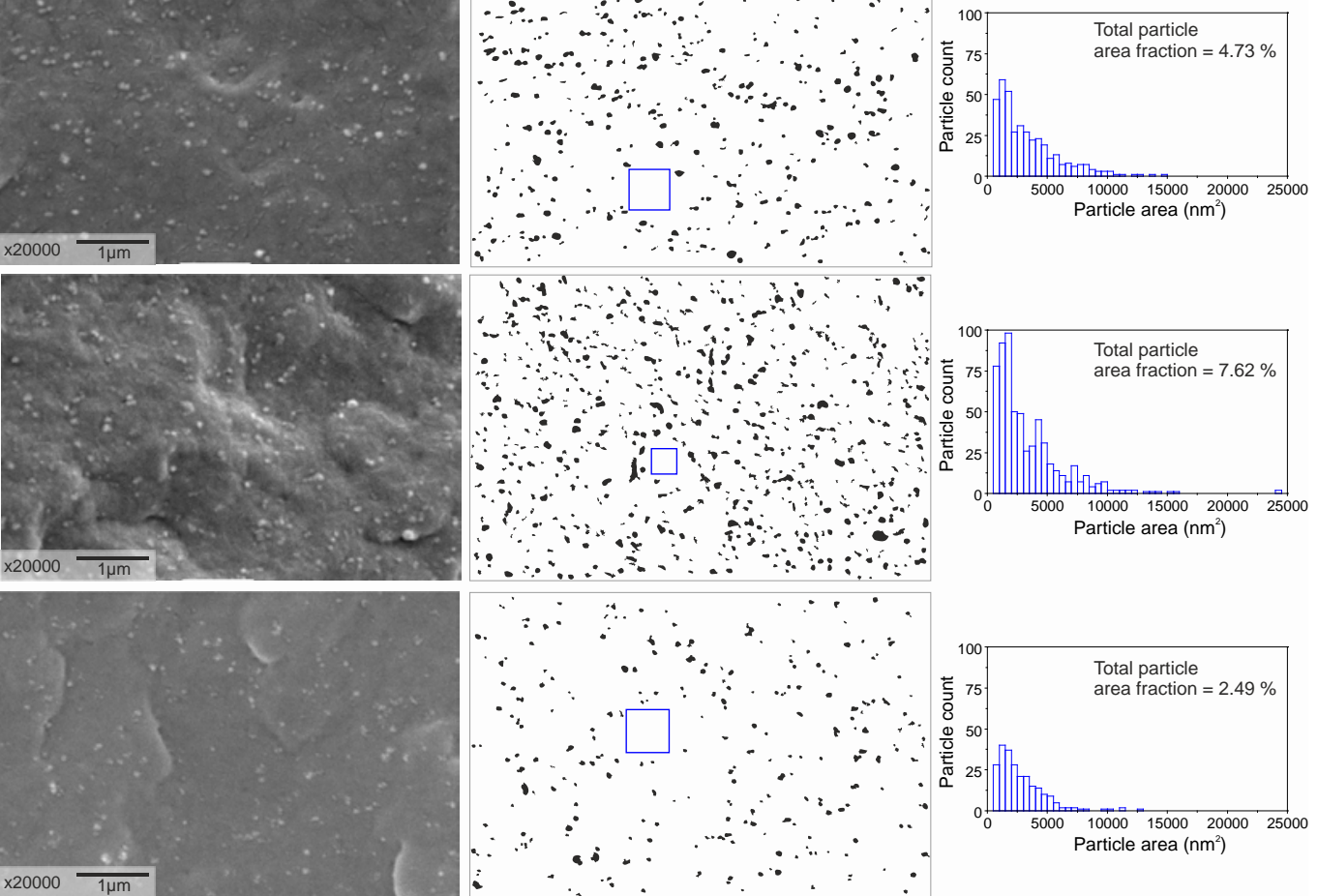

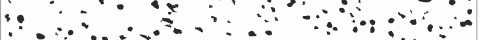
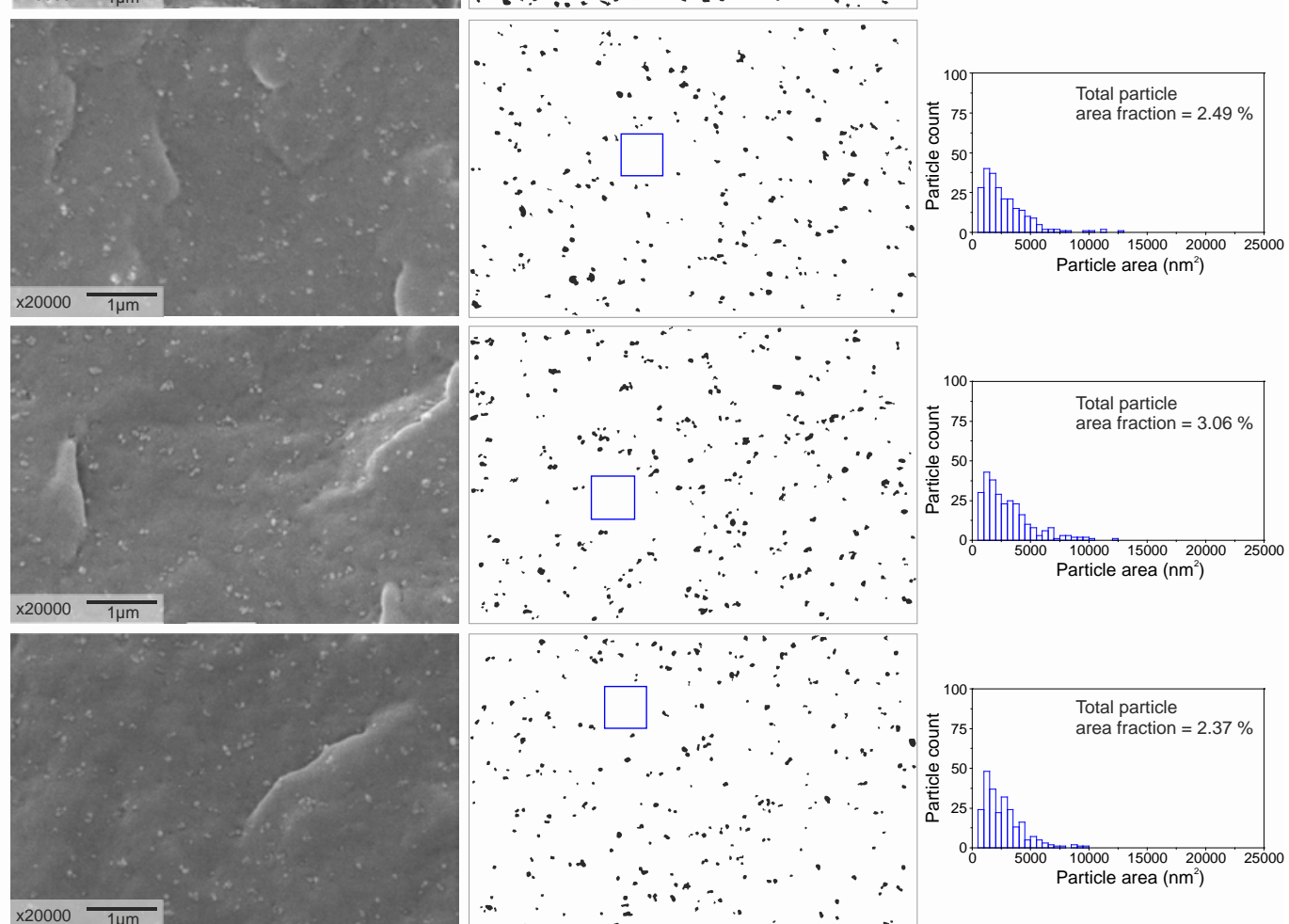

Figure 3 - SEM cross-section images (left), nanodispersion analysis (middle) and particle area histograms (right) of various 4.5 wt$\% \mathrm{SiO}_{2}$-BOPP films. The pre-mixing method, compounder details and analysis results are given in the figure. The blue boxes (middle) denote the free-space length $L_{f}$ used for the dispersion quality quantification [10]. $L_{f}$ determination was performed iteratively in MATLAB (manual mode) by gradually increasing the amount of random tests up to 100000 in order to obtain high statistical accuracy. From the particle area histograms (right), the most probable particle diameter was approximated to be $\sim 41 \mathrm{~nm}$ (assuming circular shape). The corresponding large-area multi-breakdown distributions for each film type are: (a) Figure 2d; (b) Figure 2a; (c), (d) and (e) Figure 2b. 
is sensible. This is a positive result especially when considering the cost-effectiveness and high-volume production capability of the above technology [1].

\subsection{Discussion}

The results presented above cover some of the studied processing parameters and up-scaling aspects with definite effects on the final breakdown performance of $\mathrm{SiO}_{2}$-BOPP and reference BOPP films. Other processing features with significant effects (such as the bi-axial orientation step) and less prominent effects (such as the compounder output rate) on the breakdown properties were also studied but are not presented here. Nevertheless, the previous section and the associated publications make it very clear that in addition to the compositional features (filler content, additives), the film processing stages and their optimization are in central role in achieving desirable breakdown properties with the polymer nanocomposite approach.

Aside from the material and processing related considerations alone, the breakdown measurement approach itself is also of great importance. The DC breakdown properties of the studied $\mathrm{SiO}_{2}$-BOPP PNC films have been previously shown to exhibit strong dependence on the voltage ramp rate and sample area [3]. In a more systematic study of the $4.5 \mathrm{wt}-\% \mathrm{SiO}_{2}$ BOPP films as a function of DC field-ramp-rate, an increase in Weibull shape parameter of time was observed, which points towards slow deterioration of the breakdown performance over time possibly due to space charge build-up at the PP-silica and microagglomerate interfaces [15]. Results like this emphasize the importance of careful breakdown strength assessment when dielectric films of more complex internal structure are studied, as short-term small-area breakdown tests (as defined e.g. in IEC 60243) may lead to over-optimistic results.

\section{Conclusion}

In conclusion, the large-area breakdown response of laboratory- and pilot-scale $\mathrm{SiO}_{2}$-BOPP PNC films was found to be strongly dependent on the composition and film processing. While the optimum silica content presumably resides at the low fill-fraction range ( $\sim 1$ wt$\%)$, it is not the only determining factor, as compounds with equal silica content but differences in e.g. compounder screw speed were found to exhibit large differences in the breakdown response. Indications of possible silica-antioxidant interaction were also observed. The SEM-imaging analysis of the films showed that nano-structural features cannot solely explain the observed large-area breakdown behavior this aspect points towards large-area approach being necessary for the imaging techniques as well in order to reliably establish a link between structural properties and engineering breakdown strength. Overall, the results suggest that up-scaling of PNC production is sensible with the traditional melt-blending technology, although further development and optimization of nanocomposite formulations and processing is necessary.

\section{References}

[1] J. K. Nelson, Dielectric polymer nanocomposites. Springer US, 2010.

[2] M. Takala, H. Ranta, P. Nevalainen, P. Pakonen, J. Pelto, M. Karttunen, S. Virtanen, V. Koivu, M. Pettersson, B. Sonerud, and K. Kannus, "Dielectric properties and partial discharge endurance of polypropylene-silica nanocomposite," IEEE Trans. Dielectr. Electr. Insul., vol. 17, no. 4, pp. 1259-1267, 2010.

[3] I. Rytöluoto, K. Lahti, M. Karttunen, M. Koponen, S. Virtanen, and M. Pettersson, "Large-area Dielectric Breakdown Performance of Polymer Films - Part II: Interdependence of Filler Content, Processing and Breakdown Performance in Polypropylene-Silica Nanocomposites,” IEEE Trans. Dielectr. Electr. Insul., (in press), 2015.

[4] I. Rytöluoto, K. Lahti, M. Karttunen, M. Koponen, S. Virtanen, and M. Pettersson, "Influence of low amounts of nanostructured silica and calcium carbonate fillers on the large-area dielectric breakdown performance of bi-axially oriented polypropylene,” in 2014 IEEE Conference on Electrical Insulation and Dielectric Phenomena (CEIDP), 2014, pp. 655-658.

[5] C. Calebrese, L. Hui, L. S. Schadler, and J. K. Nelson, “A review on the importance of nanocomposite processing to enhance electrical insulation," IEEE Trans. Dielectr. Electr. Insul., vol. 18, no. 4. pp. 938-945, 2011.

[6] R. Bartnikas and R. Eichhorn, Engineering Dielectrics Volume IIA Electrical Properties of Solid Insulating Materials: Molecular Structure and Electrical Behavior. Philadelphia: ASTM International, 1983.

[7] I. Rytöluoto, K. Lahti, M. Karttunen, and M. Koponen, "Largearea Dielectric Breakdown Performance of Polymer Films Part I: Measurement Method Evaluation and Statistical Considerations on Area-dependence," IEEE Trans. Dielectr. Electr. Insul., vol. 22, no. 2, pp. 689-700, 2015.

[8] I. Rytöluoto and K. Lahti, "New approach to evaluate areadependent breakdown characteristics of dielectric polymer films,” IEEE Trans. Dielectr. Electr. Insul., vol. 20, no. 3, pp. 937-946, 2013.

[9] I. Rytöluoto and K. Lahti, "Effect of Film Thickness and Electrode Area on the Dielectric Breakdown Characteristics of Metallized Capacitor Films," in 23rd Nordic Insulation Symposium (NORD-IS 13), 2013, pp. 33-38.

[10] H. S. Khare and D. L. Burris, "A quantitative method for measuring nanocomposite dispersion,” Polymer, vol. 51, no. 3, pp. 719-729, 2010.

[11] S. Virtanen, H. Ranta, S. Ahonen, M. Karttunen, J. Pelto, K. Kannus, and M. Pettersson, "Structure and dielectric breakdown strength of nano calcium carbonate/polypropylene composites," J. Appl. Polym. Sci., vol. 131, no. 1, p. 39504, 2014.

[12] J. Ho, R. Ramprasad, and S. Boggs, "Effect of alteration of antioxidant by UV treatment on the dielectric strength of BOPP capacitor film,” IEEE Trans. Dielectr. Electr. Insul., vol. 14, no. 5, pp. 1295-1301, 2007.

[13] A. Schneuwly, P. Gröning, L. Schlapbach, C. Irrgang, and J. Vogt, "Breakdown behavior of oil-impregnated polypropylene as dielectric in film capacitors," IEEE Trans. Dielectr. Electr. Insul., vol. 5, no. 6, pp. 862-868, 1998.

[14] S. Nawaz, P. Nordell, H. Hillborg, and U. W. Gedde, "Antioxidant activity in aluminium oxide - Poly(ethylene-cobutyl acrylate) nanocomposites,” Polym. Degrad. Stab., vol. 97, no. 6, pp. 1017-1025, 2012.

[15] I. Rytöluoto, M. Ritamäki, K. Lahti, and M. Karttunen, “DC Field Ramp Rate Effect on the Large-Area Multi-Breakdown Response of $\mathrm{SiO}_{2}$-BOPP Nanocomposites,” in 2015 IEEE 11th International Conference on the Properties and Applications of Dielectric Materials (ICPADM), 2015. 